\title{
Real Algebraic Realization of Characteristic Classes
}

\author{
By \\ Masahiro SHIOTA*
}

\section{§1. Introduction}

Throughout this paper $X$ denotes a real non-singular affine algebraic variety of dimension $n$. We will give a realization of the characteristic classes (the Stiefel-Whitney classes, the Pontrjagin classes and the Euler classes) of real affine algebraic vector bundles over $X$ by algebraic subvarieties (Theorems 1,2 ). For the complex field, Grothendieck [4] showed that the Chern classes of an algebraic vector bundle over a complex non-singular quasi-projective variety are realized by algebraic cycles. Morimoto [6] considered the complex analytic case. We prove Theorems 1,2 by the method used there. If we work over a real analytic vector bundle, Thom's transversality theorem shows easily a realization of the characteristic classes by analytic subsets (see Suzuki [10]).

Theorem 1 was partially proved in [2], [8], and two different applications of them were given in [2], [9].

In Section 4, Theorems 3, 4 will show that the smoothing of algebraic subvarieties of $X$ of codimension 1 for homological equivalence is always possible. The proof uses an idea in [8].

Given two cohomology classes of $X$ which are realized by algebraic subvarieties, it seems likely that their cup product is realized by an algebraic subvariety. We prove this under some assumptions, applying Theorems 1, 2 (Theorem 5). We must remark that a realization of the cup product by an analytic subset is always possible according to the transversality theorem.

Section 6 considers an affine algebraic structure of a topological vector bundle over $X$. If the rank is 1 , and if the Stiefel-Whitney class is realized by an algebraic subvariety, then the bundle has an affine algebraic structure.

Received September 20, 1981.

* Research Institute for Mathematical Sciences, Kyoto University, Kyoto, Japan. 


\section{§2. Preliminaries}

Let $V$ be an algebraic subvariety of $X$ of dimension $k$. We say that an element $\alpha \in H^{n-k}\left(X ; \mathbb{Z}_{2}\right)$ or $H^{n-k}(X ; \mathbb{Z})$ is realized by $V$ if the Poincare dual of $\alpha$ is the fundamental class $[V] \in H_{k}\left(X ; \mathbb{Z}_{2}\right)$ or $H_{k}(X ; \mathbb{Z})$ respectively where we use infinite chains if $X$ is not compact. Here, if $\alpha \in H^{n-k}(X ; \boldsymbol{Z})$ we require $X, V$ to be orientable. See Appendix in [5] and [3] for the definitions of the Poincaré dual and the fundamental class.

Let $V \subset \boldsymbol{R}^{m}, V^{\prime} \subset \boldsymbol{R}^{m^{\prime}}$ be non-singular algebraic varieties. A $C^{\infty}$ map $f$ from $V$ to $V^{\prime}$ is called smooth rational if it is the restriction of a rational map from $\boldsymbol{R}^{m}$ to $\boldsymbol{R}^{m^{\prime}}$. We call a vector bundle $V \stackrel{f}{\longrightarrow} V^{\prime}$ an affine algebraic vector bundle if the coordinate functions, the inverses and the coordinate transformations are smooth rational. Here the general linear group is provided with the natural algebraic structure, and the coordinate neighborhoods are Zariski open sets.

Let $G_{m, m^{\prime}}$ be the Grassmann manifold of $m$-linear subspaces in $\mathbb{R}^{m+m^{\prime}}$ and $V_{m, m^{\prime}}$ be the Stiefel manifold of orthogonal $m$-frames in $\boldsymbol{R}^{m+m^{\prime}}$. Then $V_{m, m^{\prime}}$ is clearly a locally closed non-singular algebraic subvariety of $\underbrace{\boldsymbol{R}^{m+m^{\prime}} \times \cdots \times \boldsymbol{R}^{m+m^{\prime}}}$. We give $G_{m, m^{\prime}}$ an algebraic structure as follows [7]. Given $T \in G_{m, m^{\prime}}$, let $P_{T}$ denote the orthogonal projection of $\boldsymbol{R}^{m+m^{\prime}}$ onto $T$. Thus $T \rightarrow P_{T}$ is an injection of $G_{m, m^{\prime}}$ into the space of linear endomorphisms of $\boldsymbol{R}^{m+m^{\prime}}$. We see easily that the image is a non-singular algebraic variety. We identify $G_{m, m^{\prime}}$ with the image. Let $p$ be the canonical map from $V_{m, m^{\prime}}$ to $G_{m, m^{\prime}}$ and $\xi_{m, m^{\prime}}: E_{m, m^{\prime}} \stackrel{q}{\longrightarrow} G_{m, m^{\prime}}$ be the vector bundle defined by

$$
E_{m, m^{\prime}}=\left\{(T, x) \in G_{m, m^{\prime}} \times \boldsymbol{R}^{m+m^{\prime}} \mid x \in T\right\} .
$$

Then $p$ is obviously smooth rational and $\xi_{m, m^{\prime}}$ is an affine algebraic vector bundle. Let $\varphi_{m, m^{\prime}}: E_{m, m^{\prime}} \rightarrow \boldsymbol{R}^{m+m^{\prime}}$ denote the projection onto the second factor.

$G_{m, m^{\prime}}$ has a cellular subdivision by the Schubert varieties (see [15]). We will be concerned with some of the varieties. Take an affine coordinate system $\left(x_{1}, \ldots, x_{m+m^{\prime}}\right)$ of $\boldsymbol{R}^{m+m^{\prime}}$, and denote by $\boldsymbol{R}^{k}$ the linear subspace defined by the equations:

$$
x_{k+1}=\cdots=x_{m+m^{\prime}}=0 \quad\left(0 \leqq k \leqq m+m^{\prime}\right) .
$$

For each integers $1 \leqq j \leqq i \leqq m$, let $F_{j}^{i} \subset G_{m, m^{\prime}}$ denote the set of all linear subspaces $T$ such that 


$$
\operatorname{dim}\left(T \cap \mathbb{R}^{m+m^{\prime}-i}\right) \geqq m+j-i .
$$

Then $F_{j}^{i}$ is an algebraic subvariety with the set of singularities $F_{j+1}^{i}$. It is wellknown [15] that $F_{1}^{i}$ realizes the $(m-i+1)$-th Stiefel-Whitney class of $\xi_{m, m^{\prime}}$.

Let $\mathfrak{m}_{k}(m, i)$ denote the set of all linear maps from $\mathbb{R}^{m}$ to $\mathbb{R}^{i}$ of rank $\leqq i-k$ for $0 \leqq k \leqq i \leqq m$. Then $\mathfrak{m}_{k}(m, i)$ is an algebraic subvariety of $\mathfrak{m}_{0}(m, i)=\mathbb{R}^{m i}$ with the set of singularities $\mathfrak{m}_{k+1}(m, i)$ for $1 \leqq k$. We remark that the stratification $\left\{\mathfrak{m}_{k}-\mathfrak{m}_{k+1}\right\}_{k=0 \ldots i}$ of $\mathfrak{m}_{0}(m, i)$ satisfies the Whitney condition A [14], namely, given $k<k^{\prime}, A \in \mathfrak{m}_{k^{\prime}}-\mathfrak{m}_{k^{\prime}+1}$ and $A_{j} \in \mathfrak{m}_{k}-\mathfrak{m}_{k+1}, j=1,2, \ldots$ such that $A_{j}$ and the tangent vector space of $\mathfrak{m}_{k}-\mathfrak{m}_{k+1}$ at $A_{j}$ tend to $A$ and an element $\tau$ in $G_{l, l^{\prime}}$ respectively for some $l, l^{\prime}$, then $\tau$ contains the tangent space of $\mathfrak{m}_{k^{\prime}}-\mathfrak{m}_{k^{\prime}+1}$ at $A$ (see [6] for a more detailed property).

Let $\eta: E \stackrel{\pi}{\longrightarrow} M$ be a $C^{\infty}$ vector bundle of rank $m$ over a $C^{\infty}$ manifold $M$. Let $f: E \rightarrow \mathbb{R}^{i}, \Phi: M \rightarrow G_{m, m^{\prime}-i}$ be $C^{\infty}$ maps such that the induced bundle $\Phi^{*}\left(\xi_{m, m^{\prime}-\imath}\right) \cong \eta$. We write the lift of $\Phi$ as $\tilde{\Phi}: E \rightarrow E_{m, m^{\prime}-i}$. Put

$$
\begin{aligned}
\Psi & =\varphi_{m, m^{\prime}-i^{\circ}} \tilde{\Phi}: E \longrightarrow \mathbb{R}^{m+m^{\prime}-i}, \\
\Psi_{f} & =(f, \Psi): E \longrightarrow \mathbb{R}^{m+m^{\prime}} .
\end{aligned}
$$

We regard $M$ as a submanifold of $E$ through the zero cross-section. Since the restriction of $\Psi_{f}$ on each fibre of $E$ is an imbedding, the image of the tangent space of the fibre at a point of $M$ under the derivative $d \Psi_{f}$ is an $m$-linear subspace in $\mathbb{R}^{m+m^{\prime}}$. This defines a $C^{\infty} \operatorname{map} \Phi_{f}: M \rightarrow G_{m, m^{\prime}}$.

A $C^{\infty} \operatorname{map} f: M \times \mathbb{R}^{m} \rightarrow \mathbb{R}^{i}$ is called in general position (at a point $a \in M$ ) if the map $\bar{f}: M \rightarrow \mathrm{m}_{0}(m, i)$ defined by

$$
\bar{f}(x)=d\left(\left.f\right|_{x \times \mathbb{R}^{m}}\right)_{x \times 0}
$$

is transversal (in a neighborhood of $a$ ) to all the strata $\mathfrak{m}_{k}(m, i)-\mathfrak{m}_{k+1}(m, i)$, $k=0, \ldots, i$. We can generalize naturally this definition to $f: E \rightarrow \mathbb{R}^{i}$ for a $C^{\infty}$ vector bundle $E$ (see [6]). The following was proved in [6].

Lemma $\mathbb{1}$. With the same notation as above, $f: E \rightarrow \mathbb{R}^{i}$ is in general position if and only if $\Phi_{f}: M \rightarrow G_{m, m^{\prime}}$ is transversal to the strata $F_{1}^{i}-F_{2}^{i}$, $F_{2}^{i}-F_{3}^{i}, \ldots, F_{i}^{i}$.

Lemma 2. If $M$ is compact, and if we provide the set of all $C^{\infty}$ maps from $E$ to $\mathbb{R}^{i}$ with the compact-open $C^{2}$ topology, then the subset of maps in general position is open and dense.

Assume that $X$ is contained in $\mathbb{R}^{n+n^{\prime}}$. Then the normal bundle of $X$ is 
equivalent to the induced bundle $h^{*} \xi_{n^{\prime}, n}$, where $h$ is the canonical smooth rational map from $X$ to $G_{n^{\prime}, n}$ defined by $h(x)=$ the space of normal vectors of $X$ at $x \in X$. The reason why $h$ is smooth rational is as follows. Let $f_{1}, \ldots, f_{n^{\prime}}$ be polynomials on $\boldsymbol{R}^{n+n^{\prime}}$ vanishing on $X$. We denote by $U$ the subset of $X$ of points at which $\operatorname{grad} f_{1}, \ldots, \operatorname{grad} f_{n^{\prime}}$ are independent, and by $v_{1}, \ldots, v_{n^{\prime}}$ the orthogonalization of Schmidt of $\operatorname{grad} f_{1}, \ldots, \operatorname{grad} f_{n^{\prime}}$ on $U$, namely,

$$
v_{1}=\operatorname{grad} f_{1}, v_{2}=\left|v_{1}\right|^{2} \operatorname{grad} f_{2}-\left(v_{1}, \operatorname{grad} f_{2}\right) v_{1}, \ldots
$$

Then the map $v(x)=\left(v_{1}, \ldots, v_{n^{\prime}}\right)$ from $U$ to $V_{n^{\prime}, n}$ is smooth rational and satisfies the equation $p \circ v=h$ on $U$. As $X$ is covered by such $U$ for suitable $f_{1}, \ldots, f_{h^{\prime}}, h$ is smooth rational on $X$. This shows also that given $V \stackrel{\pi}{\longrightarrow} X$ an affine algebraic vector bundle of rank $m$, there exists a smooth rational map $g: X \rightarrow G_{m, m^{\prime \prime}}$ for some $m^{\prime \prime}$ such that $g^{*} \xi_{m, m}$ " is rationally equivalent to $V \stackrel{\pi}{\longrightarrow} X$. Moreover it follows that for any smooth rational map $f: V \rightarrow \mathbb{R}^{i}, g_{f}: X \rightarrow G_{m, m^{\prime}}$ is smooth rational with $m^{\prime}=m^{\prime \prime}+i$, and $g_{f}^{*} \xi_{m, m^{\prime}}$ is also rationally equivalent to $V \stackrel{\pi}{\longrightarrow} X$.

Lastly we need the concept of stratified map. Let $h$ be a smooth rational map from $X$ to another non-singular affine algebraic variety $X^{\prime}$. Then we can stratify $h$, that is, there exist non-singular semi-algebraic subvarieties $Y_{1}, \ldots, Y_{k}$ of $X^{\prime}$ such that $X^{\prime}$ is the disjoint union of $Y_{1}, \ldots, Y_{k}$, that $Z_{j}=h^{-1}\left(Y_{j}\right)$ is nonsingular for each $j$, that the restriction of $h$ on $Z_{j}$ is a submersion to $Y_{j}$ for each $j$, and that the stratification $\left\{Y_{j}\right\}_{j}$ satisfies the Whitney condition A. For the proof, see [14].

A $C^{\infty}$ function is called regular at a point if one of the first partial derivatives takes a non-zero value at the point.

\section{§3. Algebraic Realization of Characteristic Classes}

Theorem 1. The Stiefel-Whitney classes of any affine algebraic vector bundle over $X$ are realized by algebraic subvarieties. We can choose a nonsingular algebraic subvariety of realization of the $k$-th class for $k=1, k \geqq$ $(n-1) / 2$ or $k=$ rank of the bundle.

Theorem 2. Assume that $X$ is orientable. Then the Pontrijagin classes of an affine algebraic vector bundle over $X$ are realized by orientable algebraic subvarieties. If moreover the bundle is orientable, this statement holds for the Euler class. The subvarieties of realizations of the Eular class and the $k$-th Pontrjagin class can be non-singular, for $k \geqq(n-2) / 6$. 
Proof of Theorem 1. Let $m$ be the rank of the bundle, and $1 \leqq i \leqq m$ be an integer. We want to realize the $(m-i+1)$-th Stiefel-Whitney class by an algebraic subvariety. Through the argument in Section 2, we regard the bundle as the induced bundle $h^{*} \zeta_{m, i h^{\prime}-i}$ for a smooth rational map $h: X \rightarrow G_{m, m^{\prime}-i}$. Assume that there exists a smooth rational map $f: h^{*} E_{m, m^{\prime}-i} \rightarrow \mathbb{R}^{i}$ in general position. Then, by Lemma $1 h_{f}: X \rightarrow G_{m, m^{\prime}}$ is transversal to the strata $F_{1}^{i}$ $-F_{2}^{i}, F_{2}^{i}-F_{3}^{i}, \ldots$. Now $F_{1}^{i}$ realizes the $(m-i+1)$-th Stiefel-Whitney class of $\xi_{m, m^{\prime}}$. Hence $h_{f}^{-1}\left(F_{1}^{i}\right)$ is an algebraic subvariety of realization. Thus it is sufficient to show that at least one smooth rational map $f: h^{*} E_{m, m^{\prime}-i} \rightarrow \mathbb{R}^{i}$ is in general position. This is immediately proved by Lemma 2 if $X$ is compact.

By the remark at the end of Section 2, there exist non-singular semi-algebraic subvarieties $Y_{1}, \ldots, Y_{l}$ of $G_{m, m^{\prime \prime}}$ with $m^{\prime \prime}=m^{\prime}-i$ such that $G_{m, m^{\prime \prime}}$ is the disjoint union of $Y_{1}, \ldots, Y_{l}$, that $Z_{j}=h^{-1}\left(Y_{j}\right)$ is non-singular for each $j$, that $\left\{Y_{j}\right\}$ satisfies the Whitney condition $\mathrm{A}$, that $\left.h\right|_{Z_{i}}$ is a submersion to $Y_{j}$ for each $j$, and that $\operatorname{dim} Y_{j} \leqq \operatorname{dim} Y_{j^{\prime}}$ for $j \leqq j^{\prime}$. Let $\check{\zeta}_{j}: E_{j} \stackrel{q}{\longrightarrow} Y_{j}$ denote the restriction of $\xi_{m, m^{\prime \prime}}$ on $Y_{j}$ for $j=1, \ldots, l$.

Now we want to find a $C^{\infty}$ map $g: E_{m, m^{\prime \prime}} \rightarrow \mathbb{R}^{i}$ such that the restriction of $g$ on $E_{j}$ is in general position for $j=1, \ldots, l$. Since $Y_{1}$ is compact, by Lemma 2 there exists a $C^{\infty}$ map $g_{1}: E_{1} \rightarrow \mathbb{R}^{i}$ in general position. Let $g_{1}^{\prime}$ be a $C^{\infty}$ extension of $g_{1}$ on $E_{m, m^{\prime \prime}}$.

The restriction $\left.g_{1}^{\prime}\right|_{E_{2}}$ is in general position on an open neighborhood $U_{1}$ of $Y_{1}$ in $G_{m, m}$. The reason is the following. As the problem is local, we can assume $E_{m, m^{\prime \prime}}=G_{m . m^{\prime \prime}} \times \mathbb{R}^{m}$. Assume that there does not exist such $U_{1}$. Then we have a sequence of points $a_{1} a_{2}, \ldots$, in $Y_{2}$ such that

$$
\overline{\left.g_{1}^{\prime}\right|_{E_{2}}}=\left.\bar{g}_{1}^{\prime}\right|_{Y_{2}}: Y_{2} \longrightarrow \mathrm{m}_{0}(m, i)
$$

is not in general position at $a_{1}, a_{2}, \ldots$, and that $a_{1}, a_{2}, \ldots$ tend to a point $b$ of $Y_{1}$. We write briefly $m_{j}(m, i)=m_{j}$. Choosing a subsequence, we can assume that $c_{j}=\bar{g}_{1}^{\prime}\left(a_{j}\right), j=1,2, \ldots$ are all contained in one $m_{k}-m_{k+1}$ for some $k$, and that the tangent vector space $\alpha_{j}$ of $Y_{2}$ at $a_{j}$ and $\gamma_{j}$ of $\mathfrak{m t}_{k}-\mathfrak{m}_{k+1}$ at $c_{j}, j=1,2, \ldots$ tend to $\alpha \in G_{l, l^{\prime}}$ and $\gamma \in G_{l^{\prime \prime}, m_{\mathrm{s}}-l^{\prime \prime}}$ respectively for some $l, l^{\prime}, l^{\prime \prime}$. Then, by the Whitney condition $\mathrm{A}$ on $\left\{Y_{j}\right\}$ and $\left\{\mathfrak{n}_{j}-\mathfrak{m}_{j+1}\right\}, \alpha$ and $\gamma$ contain the tangent vector spaces $\beta$ of $Y_{1}$ at $b$ and $\delta$ of $\mathfrak{m}_{k^{\prime}}-\mathfrak{m}_{k^{\prime}+1}$ at $d=\bar{g}_{1}^{\prime}(b)$ respectively where $d \in \mathfrak{m}_{k^{\prime}}-\mathfrak{m}_{k^{\prime}+1}$. Since $\left.\bar{g}_{1}^{\prime}\right|_{Y_{1}}$ is transversal to $m_{k^{\prime}}-m_{k^{\prime}+1}$ at $b$, we have

$$
d\left(\bar{g}_{1}^{\prime}\right)_{b}(\beta)+\delta=T_{d} \text { the tangent vector space of } m_{0} \text { at } d .
$$

Hence 


$$
d\left(\bar{g}_{1}^{\prime}\right)_{b}(\alpha)+\gamma=T_{d} .
$$

It follows that $d\left(\bar{g}_{1}^{\prime}\right)_{a_{j}}\left(\alpha_{j}\right)+\gamma_{j}=T_{a_{j}}$ for large $j$. This means that $\left.\bar{g}_{1}^{\prime}\right|_{Y_{2}}$ is transversal to $\mathfrak{n t}_{h}-\mathfrak{m}_{h+1}$ at $a_{j}$. This is a contradiction. Thus $\left.g_{1}^{\prime}\right|_{E_{2}}$ is in general position on some $U_{1}$.

Let $W_{1}$ be an open neighborhood of $Y_{1}$ in $G_{m, m^{\prime \prime}}$ whose closure is contained in $U_{1}, E_{2}^{\prime}$ denote the restriction of $E_{2}$ on $Y_{2}-W_{1}$. By Lemma 2 we can approximate $\left.g_{1}^{\prime}\right|_{E_{2}^{\prime}}$ by a $C^{\infty}$ map $g_{2}$ in general position on $E_{2}^{\prime}$. Let $\psi$ be a $C^{\infty}$ function defined on $G_{m, m^{\prime \prime}}$ such that

$$
\psi=\left\{\begin{array}{lll}
1 & \text { on } & W_{1} \\
0 & \text { on } & Y_{2}-U_{1}
\end{array}\right.
$$

Write

$$
g_{2}^{\prime}(x)=\psi \circ q(x) g_{1}^{\prime}(x)-(1-\psi) \circ q(x) \times g_{2}(x), \quad x \in E_{1} \cup E_{2} .
$$

Then, taking a closer approximation $g_{2}$, we can assume that $\left.g_{2}^{\prime}\right|_{E_{1}}=g_{1}$ and $\left.g_{2}^{\prime}\right|_{E_{2}}$ are in general position. Let $g_{2}^{\prime \prime}$ be a $C^{\infty}$ extension of $g_{2}^{\prime}$ on $E_{m, m^{\prime \prime}}$. We repeat this argument on $g_{2}^{\prime \prime}, E_{3}, \ldots$. Then we have a $C^{\infty}$ map $g: E_{m, m^{\prime \prime}} \rightarrow \mathbb{R}^{i}$ such that $\left.g\right|_{E_{j}}$ is in general position for $j=1, \ldots, l$.

Moreover the argument above implies that the set of such maps $g$ is open and dense in the set of $C^{\infty}$ maps from $E_{m, m^{\prime \prime}}$ to $\boldsymbol{R}^{i}$. Therefore there exists a smooth rational map $g: E_{m, m^{\prime \prime}} \rightarrow \boldsymbol{R}^{i}$ such that $\left.g\right|_{E_{J}}$ is in general position for $j=1, \ldots, l$.

For each $j=1, \ldots, l$, consider $f_{j}$ the restriction of $f=g \circ \tilde{h}$ on $\tilde{Z}_{j}=\tilde{h}^{-1}\left(E_{j}\right)$ where $\tilde{h}: h^{*} E_{m, m^{\prime \prime}} \rightarrow E_{m, m^{\prime \prime}}$ is the lift of $h$. Then we see that $f_{j}$ is in a general position as follows. As the problem is local, we can assume

$$
E_{j}=Y_{j} \times \boldsymbol{R}^{m}, \tilde{Z}_{j}=Z_{j} \times \boldsymbol{R}^{m}, \quad \tilde{h}(z, v)=(h(z), v) \quad \text { for } \quad(z, v) \in Z_{j} \times \boldsymbol{R}^{m} .
$$

Then we have

$$
\bar{f}_{j}=\left.\bar{g}\right|_{Y_{j}},\left.h\right|_{Z_{j}}: Z_{j} \longrightarrow \mathfrak{m} \mathfrak{m}_{0} .
$$

We saw already that $\left.h\right|_{Z_{j}}$ is a submersion to $Y_{j}$ and that $\left.\bar{g}\right|_{Y_{j}}: Y_{j} \rightarrow \mathfrak{m}_{0}$ is transversal to all strata $m_{1}-\mathfrak{m}_{2}, m_{2}-m_{3}, \ldots$. Hence $\bar{f}_{i}$ is transversal to $\mathfrak{m}_{1}-m_{2}$, $\ldots$, namely $f_{j}$ is in general position. Furthermore it follows that $f$ is in general position at any point of $Z_{j}$, hence globally. Thus we have constructed an algebraic realization of the Stiefel-Whitney classes.

As the codimension of $F_{2}^{i}$ (the set of singular points of $F_{1}^{i}$ ) in $G_{m, m^{\prime}}$ is $2(m-i+2), h_{f}^{-1}\left(F_{2}^{i}\right)$ is of dimension at most $n-2(m-i+2)$. Hence the above 
algebraic subvariety of realization of the $k$-th Stiefel-Whitney class is nonsingular for $k \geqq(n-1) / 2$. Since $F_{1}^{1}$ is non-singular, the realization of $m$-th Stiefel-Whitney class is non-singular.

A smoothing of the algebraic realization of the first Stiefel-Whitney class follows from Theorem 3 below, because the subvariety is of codimension 1 . Thus the theorem is proved.

Proof of Theorem 2. We prove this in the same way as above. For the Pontrjagin classes, we only have to choose $m^{\prime \prime}$ so that $m+m^{\prime}$ is even. Then it is well-known [15] that $G_{m, m^{\prime}}$ and $F_{2}^{m-2 i+2}$ are orientable, and that $F_{2}^{m-2 i+2}$ realizes the $i$-th Pontrjagin class of $\xi_{m, m^{\prime}}$. Hence $h_{f}^{-1}\left(F_{2}^{m-2 i+2}\right)$ is an algebraic realization of the $i$-th Pontrjagin class of $h_{f}^{*} \xi_{m, m^{\prime}}$. Furthermore $h_{f}^{-1}\left(F_{2}^{m-2 i+2}\right)$ is non-singular for $i \geqq(n-2) / 6$, because the set of singular points of $F_{2}^{m-2 i+2}$ is $F_{3}^{m-2 i+2}$, and the codimension of $F_{3}^{m-2 i+2}$ is $6 i+3$.

For the Euler class, choose $m^{\prime \prime}$ so that $m+m^{\prime}$ is odd. Let $\widetilde{G}_{m, m^{\prime}}$ denote the oriented Grassmann manifold, and $p: \widetilde{G}_{m, m^{\prime}} \rightarrow G_{m, m^{\prime}}$ denote the natural projection. Then $p$ is a 2-fold covering. Since the bundle in problem is orientable, there exists a $C^{\infty}$ map $\tilde{h}_{f}: X \rightarrow \widetilde{G}_{m, m^{\prime}}$ such that $p \circ \tilde{h}_{f}=h_{f}$. We know (see [15]) that the Euler class of $p^{*} \xi_{m, m^{\prime}}$ is realized by $p^{-1}\left(F_{1}^{1}\right)$. Here we must remark that $\widetilde{G}_{m, m^{\prime}}$ and $F_{1}^{1}$ are orientable. Hence $h_{f}^{-1}\left(F_{1}^{1}\right)$ realizes the Euler class of $\xi_{m, m^{\prime}}$. This subvariety is non-singular, because $F_{1}^{1}$ is non-singular. Thus we have proved the theorem.

Remark 1. Consider the case of tangent bundle in Theorem 1. Let $1 \leqq k \leqq n$ be an odd integer or equal to $n$. The $k$-th Stiefel-Whitney class of the bundle is an element of $H^{k}(X ; \mathbb{Z})$ where we use twisted coefficients. The Poincaré dual of this class is a homology class $W_{k}$ of $X$ with coefficients in $\mathbb{Z}$. Here we use infinite chain if $X$ is not compact. The algebraic subvariety of realization of the $k$-th Stiefel-Whitney class which was constructed in the proof of Theorem 1 is orientable and has the oriented fundamental class $W_{k}$.

Remark 2. Even if a cohomology class of $X$ is realized by a $C^{\infty}$ manifold, it is not necessarily realized by an algebraic subvariety. See an example in [1].

\section{§4. Smoothing of Algebraic Subvarieties}

Given algebraic subvarieties $V, V^{\prime}$ of $X$, we call them $\mathbb{Z}_{2}$-homologically 
equivalent if their fundamental classes are identical. If the fundamental classes have the coefficients in $\mathbb{Z}$, then $V$ and $V^{\prime}$ are called $\mathbb{Z}$-homologically equivalent. Moreover, " $Z$-(or $\mathbb{Z}_{2^{-}}$) smoothing $V$ " means that we can find a non-singular $V^{\prime}$.

Theorem 3. The $\mathbb{Z}_{2}$-smoothing of any algebraic subvariety $V$ of $X$ of codimension 1 is possible. Assume moreover that $X$ is compact. Let $M$ be a $C^{\infty}$ submanifold of $X \boldsymbol{Z}_{2}$-homologically equivalent to $V$. Then we can $\boldsymbol{Z}_{2}$-smooth $V$ so that the smoothed subvariety is the image of $M$ under a $C^{\infty}$ diffeomorphism of $X$ arbitrarily close to the identity.

Theorem 4. Assume that $X$ is compact. Let $V, M$ be an orientable algebraic subvariety and an orientable $C^{\infty}$ submanifold respectively which are $\boldsymbol{Z}$-homologically equivalent. Then we can $\boldsymbol{Z}$-smooth $V$ so that the smoothed subvariety is the image of $M$ under a $C^{\infty}$ diffeomorphism of $X$ arbitrarily close to the identity. Especially, if $X$ is compact and orientable, the $\mathbb{Z}$ smoothing of any orientable algebraic subvariety of codimension 1 is possible.

Proofs. The first half of Theorem 4 follows trivially from the latter half of Theorem 3. The latter half of Theorem 4 is an immediate consequence of the first half of Theorem 4 and the fact that any first $\mathbb{Z}$-cohomology class of a compact orientable manifold is realized by a $C^{\infty}$ submanifold (see [11]). Hence we need only to prove Theorem 3 .

Let $X^{\prime}$ be the desingularization of Hironaka of the algebraic closure of $X$ in $P^{n^{\prime}}(\boldsymbol{R})$ where $X \subset \boldsymbol{R}^{n^{\prime}}$. Let $V^{\prime}$ be the algebraic closure of $V$ in $X^{\prime}$. If we can smooth $V^{\prime}$, the intersection of the smoothed subvariety and $X$ is non-singular and $\boldsymbol{Z}_{2}$-homologically equivalent to $V$. Hence, from the beginning we can assume that $X$ is compact. Then the first half of Theorem 3 follows from the latter half and the fact that any first $\mathbb{Z}_{2}$-cohomology class of a compact manifold is realized by a $C^{\infty}$ submanifold [11]. Thus it is sufficient to prove the latter half of Theorem 3. Obviously we can assume that $M$ is analytic.

If an irreducible component of $V$ is of codimension $>1$, we can remove it, because the removing does not affect the fundamental class of $V$. Hence we assume that all irreducible components of $V$ are of codimension 1. Moving $M$ if necessary, we can assume also that the germs of $M$ and of $V$ at each point of $M \cup V$ are not identical. Let $\Gamma, \mathcal{O}$ denote the rings of smooth rational functions, analytic functions on $X$ respectively. Let $\mathfrak{a} \subset \Gamma, \mathfrak{b} \subset \mathcal{O}$ be the ideals of functions vanishing on $V, M$ respectively.

Now we will prove in the same way as Lemma 1 in [8] that $\mathfrak{c}=\mathfrak{a} \mathcal{O} \cap \mathfrak{b}$ is a 
principal ideal. Let $K$ be a triangulation of $X, K^{\prime}$ be a subcomplex such that $\left|K^{\prime}\right|=V \cup M$ and that each $(n-2)$-simplex of $K^{\prime}$ is faces of even-numbered $(n-1)$ simplexes of $K$. Put

$$
\begin{aligned}
& \Gamma_{x}=\{f / g \mid f, g \in \Gamma, g(x) \neq 0\} \text { for } x \in X, \\
& \mathcal{O}_{x}=\text { the ring of analytic function germs at } x \in X .
\end{aligned}
$$

We write

$$
\mathfrak{a}_{x}=\mathfrak{a} \Gamma_{x}, \mathfrak{b}_{x}=\mathfrak{b} \mathcal{O}_{x} \text { and } \mathfrak{c}_{x}=\mathfrak{c} \mathcal{O}_{x} .
$$

By the assumption on the irreducible components of $V, \mathfrak{a}$ is the intersection of prime ideals of height 1 , hence so is $\mathfrak{a}_{x}$ for any $x \in X$. Clearly $\mathfrak{b}$ and $\mathfrak{b}_{x}, x \in X$, have the same property. Since $X$ is non-singular, $\Gamma_{x}$ and $\mathcal{O}_{x}$ are unique factorization rings for any $x \in X$. Therefore $\mathfrak{a}_{x}, \mathfrak{b}_{x}$ and hence $\mathfrak{c}_{x}, x \in X$, are principal ideals, and we have

$$
\mathfrak{c}_{x}=\mathfrak{a}_{x} \mathfrak{b}_{x}
$$

Here we remark that $\mathfrak{b}_{x}$ is the set of analytic function germs vanishing on $M$ (Cartan Theorem A on Stein manifold).

Let $\Delta_{1}, \ldots, \Delta_{l}$ be all the n-simplexes of $K$. We can suppose the existence of $f_{1}, \ldots, f_{l}$ in $\mathfrak{c}$ such that for each $i, f_{i}$ is a generator of $\mathfrak{c}_{x}$ if $x$ is a point of the star of $\Delta_{i}$. Let $s_{1}, \ldots, s_{r}$ be all the $(n-1)$-simplexes of $K^{\prime}$. Then the chain $s_{1}+\cdots$ $+s_{r}$ is a cycle. By the hypotheses, this is the boundary of a chain, say, $\Delta_{1}+\cdots$ $+\Delta_{l^{\prime}}, l^{\prime}<l$. We choose $f_{i}$ so that $f_{i}$ is positive in the interior of $\Delta_{i}$ if $i \leqq l^{\prime}$, and negative if $i>l^{\prime}$. Then, for each $\Delta_{i}$ and each $\Delta_{j}$ in $\operatorname{St}\left(\Delta_{i}\right), f_{i}$ and $f_{j}$ take the same sign in $\Delta_{j}$, and $f_{j} / f_{i}$ is positive and smooth there, because, if $s_{k}$ is the common face of $\Delta_{i}$ and $\Delta_{j}, s_{k}$ contains non-singular points of $V$, hence the sign of values of $f_{i}$ changes through $s_{k}$.

Let $\rho_{i}, i=1, \ldots, l$ be elements of $\Gamma$ such that $0 \leqq \rho_{i}$, and $\rho_{i} \geqq 1$ on $\Delta_{i}$ and $\leqq \varepsilon$ outside of $\operatorname{St}\left(\Delta_{i}\right)$ where $\varepsilon$ is a small positive number. We choose $\varepsilon$ so small that for each $\Delta_{i}$ and each $\Delta_{j} \not \subset \operatorname{St}\left(\Delta_{i}\right), \rho_{j} f_{j} / f_{i}$ is close to the zero function on $\Delta_{i}$. Then the $\operatorname{sum} f$ of $\rho_{i} g_{i}, i=1, \ldots, l$, generates $\mathfrak{c}_{x}$ for all $x$ of $X$. Hence $f$ is a generator of $\mathfrak{c}$, namely, $\mathfrak{c}$ is a principal ideal.

Let $f$ be a generator of $\mathfrak{c}$, and $g_{1}, \ldots, g_{k}$ be a system of generators of $\mathfrak{a}$. Then we have analytic functions $\varphi_{1}, \ldots, \varphi_{k}$ defined on $X$ such that

$$
f=\varphi_{1} g_{1}+\cdots+\varphi_{k} h_{k} .
$$

Let $\psi_{1}, \ldots, \psi_{k}$ be approximations of $\varphi_{1}, \ldots, \varphi_{k}$ by smooth rational functions. Put 


$$
\begin{aligned}
f^{\prime} & =\psi_{1} g_{1}+\cdots+\psi_{k} g_{k}, \\
M^{\prime} & =\text { the closure of } f^{\prime-1}(0)-V .
\end{aligned}
$$

Then $M^{\prime}$ is a non-singular algebraic subvariety and can be translated to $M$ by a $C^{\infty}$ diffeomorphism of $X$ close to the identity. The reason is the following. Since $\mathfrak{a}_{x}$ is a principal ideal for each $x \in X$, there exists a closed neighborhood $U$ of $x$ where one of $g_{1}, \ldots, g_{k}$, say $g_{1}$, is a generator. Hence we have rational functions $h_{2}, \ldots, h_{k}$ on $X$ smooth on $U$ such that $g_{i}=h_{i} g_{1}, i=2, \ldots, k$. Put

$$
\Phi_{U}=\varphi_{1}+\varphi_{2} h_{2}+\cdots+\varphi_{k} h_{k} \quad \text { on } U \text {. }
$$

It follows that $f=\Phi_{U} g_{1}$ on $U$. By $(*), \Phi_{U}$ is a generator of $\mathfrak{b}_{x^{\prime}}$ for any $x^{\prime} \in U$. This means that the set of zero points of $\Phi_{U}$ is $U \cap M$ and that $\Phi_{U}$ is regular at $U \cap M$. Clearly

$$
\Phi_{U}=\psi_{1}+\psi_{2} h_{2}+\cdots+\psi_{k} h_{k} \quad \text { on } U
$$

is an approximation of $\Phi_{U}$ by a smooth rational function, and we have

$$
\Phi_{U}^{-1}(0)=U \cap M^{\prime} .
$$

Choose a close approximation, and cover $X$ by such $U$ 's. Then we can assume that

$$
U \cap M=\phi \quad \text { or } \quad \tau:(U, U \cap M) \stackrel{\leftrightharpoons}{\leftrightharpoons}\left([0,1]^{n}, 0 \times[0,1]^{n-1}\right) .
$$

If $U \cap M=\phi$, we have $U \cap M^{\prime}=\phi$. In the second case, we can assume moreover that the union $W$ of $U$ 's such that $U \cap M \neq \phi$ is a tubular neighborhood of $M$, that the projection $\pi$ of $W$ onto $M$ corresponds to

$$
\pi^{\prime}:[0,1]^{n} \ni\left(z_{1}, \ldots, z_{n}\right) \longrightarrow\left(0, z_{2}, \ldots, z_{n}\right) \in 0 \times[0,1]^{n-1},
$$

and that $\Phi_{U^{\circ}} \tau^{-1}=z_{1}$. Then $\Psi_{U^{\circ}} \tau^{-1}$ is an approximation of $z_{1}$. Hence $\Psi_{U^{\circ}} \tau^{-1}$ is a regular function, and $\tau\left(U \cap M^{\prime}\right)$ (=the set of zero points) is translated to $0 \times[0,1]^{n-1}$ by $\pi^{\prime}$. Thus $\left.\pi\right|_{M^{\prime}}$ is a diffeomorphism from $M^{\prime}$ to $M$. Obviously it is possible to extend $\left.\pi\right|_{M^{\prime}}$ onto $X$. It is easy to see that the closer the approximation of $\varphi_{1}, \ldots, \varphi_{k}$ is, the more the extension of $\left.\pi\right|_{M}$, is chosen close to the identity. The argument above shows also that $M^{\prime}$ is a non-singular algebraic subvariety; hence the theorem is proved.

Let $V, V^{\prime}$ be non-singular algebraic subvarieties of $X$, and $\alpha, \alpha^{\prime}$ denote the cohomology classes realized by $V, V^{\prime}$. If $V$ and $V^{\prime}$ intersect transversally, the cup product of $\alpha$ and $\alpha^{\prime}$ is realized by $V \cap V^{\prime}$. Hence we can generalize Theorems 3, 4 as follows. 
Theorem 3'. Let a cohomology class $\alpha \in H^{k}\left(X ; \mathbb{Z}_{2}\right), k=1, \ldots, n$, be the cup $k$-product of the first cohomology classes $\beta_{1}, \ldots, \beta_{k}$. If $\beta_{1}, \ldots, \beta_{k}$ are realized by algebraic subvarieties, then $\alpha$ is realized by a non-singular algebraic subvariety.

Theorem 4'. Assume that $X$ is orientable and compact. The above statement holds for $\mathbb{Z}$-coefficients.

Example. From Theorems 1 and $3^{\prime}$, it follows that if $X$ is homeomorphic to $P^{n}(\boldsymbol{R}), n$ even, any $\mathbb{Z}_{2}$-cohomology class of $X$ is realized by a non-singular algebraic subvariety.

\section{§5. Cup Product of Realizable Cohomology Classes}

Let $V, V^{\prime}$ be algebraic subvarieties of $X$ of codimension $k, k^{\prime}$. Let $\alpha, \alpha^{\prime}$ be the cohomology classes realized by $V, V^{\prime}$ respectively, and $\left\{Y_{i}\right\},\left\{Y_{j}^{\prime}\right\}$ be respective stratifications of $V, V^{\prime}$. If each $Y_{i}$ and each $Y_{j}^{\prime}$ intersect transversally, then the cup product $\alpha \cup \alpha^{\prime}$ is realized by the intersection $V \cap V^{\prime}$. In this section we will weaken these conditions. The coefficient ring may be $\mathbb{Z}_{2}$ or $\mathbb{Z}$. For $\mathbb{Z}$, we must remember the arrangement at the beginning of Section 2 .

Put $V^{\prime \prime}=V \cap V^{\prime}$. Let $V_{s}, V_{s}^{\prime}$ and $V_{s}^{\prime \prime}$ denote the respective sets of singular points of $V, V^{\prime}$ and $V^{\prime \prime}$. Let $T$. denote the tangent vector space.

Theorem 5. Let an algebraic subvariety $S$ satisfy the following properties

(i) $S \supset V^{\prime \prime} \cap\left(V_{s} \cup V_{s}^{\prime} \cup V_{s}^{\prime \prime}\right)$,

(ii) $\operatorname{codim} S>k+k^{\prime}$,

(iii) $T R=\left.\left.T\left(V-V_{s}\right)\right|_{R} \cap T\left(V^{\prime}-V_{s}^{\prime}\right)\right|_{R}$ with $R=V^{\prime \prime}-S$.

Then $\alpha \cup \alpha^{\prime}$ is realized by an algebraic subvariety, and the set of singular points of the subvariety can be contained in $S$.

Proof. At first we suppose $S=\phi$. Then any point of $V^{\prime \prime}$ is a non-singular point of $V$ and $V^{\prime}$. Since $V^{\prime \prime}$ is non-singular, any irreducible component is a union of connected components. Hence, by the argument below we can assume that $V^{\prime \prime}$ is a manifold of dimension $n^{\prime}$. Put $m=n+n^{\prime}-k-k^{\prime}$. Let $\xi: Y \stackrel{\tau}{\longrightarrow} V^{\prime \prime}$ be the subbundle of $\left.T X\right|_{R}$ of vectors orthogonal to $\left.\left.T V\right|_{V^{\prime \prime}} \oplus T V^{\prime}\right|_{V^{\prime \prime}}$. We saw in Section 2 that the normal bundle of $X$ is an affine algebraic bundle. By the same method it follows that $\xi$ is an affine algebraic bundle. Let $f$ be a $C^{\infty}$ cross-section of $\xi$ such that $f\left(V^{\prime \prime}\right)$ and $V^{\prime \prime}$ intersect transversally. Here we identify $V^{\prime \prime}$ with its image under the zero cross-section of $\xi$. We write the intersection as $Z$. 
Using $f$, we can construct a $C^{\infty}$ diffeomorphism $\theta$ of $X$ arbitrarily close to the identity in the $C^{x}$ Whitney topology such that $\theta(x)=x$ for any singular point $x$ of $V$ and $V^{\prime}$, that $V$ and $\theta\left(V^{\prime}\right)$ intersect transversally and that $V \cap \theta\left(V^{\prime}\right)=Z$. Hence the $C^{\infty}$ manifold $Z$ realizes $\alpha \cup \alpha^{\prime}$. On the other hand, by Lemma 3 below, $Z$ realizes the $m$-th Stiefel-Whitney class of $\zeta$. Apply Theorem 1 to $\xi$. Then it follows that $\alpha \cup \alpha^{\prime}$ is realized by a non-singular algebraic subvariety. If the coefficient ring is $\mathbb{Z}$, it is sufficient to treat the Euler class of $\xi$ instead of the Stiefel-Whitney class.

The case $S \neq \phi$. Apply the result above to $X-S, V-S$ and $V^{\prime}-S$. We remark that the algebraic varieties are affine. Let $Z \subset X-S$ be an algebraic subvariety of realization of $\left.\left(\alpha \cup \alpha^{\prime}\right)\right|_{X-S}, Z^{\prime}$ be the algebraic closure of $Z$ in $X$. Then $Z^{\prime}$ is an algebraic subvariety of realization of $\alpha \cup \alpha^{\prime}$ because of $\operatorname{dim}\left(Z^{\prime} \cap S\right)$ $<\operatorname{dim} Z^{\prime}$. Hence we proved the theorem.

Example. Assume that $V, V^{\prime}$ are non-singular. If $V \supset V^{\prime}$, the conditions in Theorem 5 are satisfied.

Let $\eta: E \stackrel{\pi}{\longrightarrow} M$ be a $C^{\infty}$ vector bundle of rank $m$ over a $C^{\infty}$ manifold $M$. Let $f$ be a $C^{\infty}$ cross-section of $\eta$. Then we have

Lemma 3. Assume that the image $f(M)$ and $M$ intersect transversally. Then the intersection realizes the $m$-th Stiefel-Whitney class of $\eta$. Furthermore it realizes the Euler class if $M$ and $\eta$ are orientable.

Proof. We use the notation in Section 2. First, consider the case $\eta$ $=\zeta_{m, m^{\prime}}$. Then $F_{1}^{1}$ realizes the $m$-th Stiefel-Whitney class of $\xi_{m, m^{\prime}}$. Hence it is sufficient to find a $C^{\infty}$ cross-section $f$ of $\xi_{m, m^{\prime}}$ such that $f\left(G_{m, m}\right)$ intersects transversally with $G_{m, m}$ at $F_{1}^{1}$. Put

$$
f(T)=P_{T}((0, \ldots, 0,1)) \quad \text { for } \quad T \in G_{m, m^{\prime}} .
$$

Then, obviously $f\left(G_{m, m^{\prime}}\right) \cap G_{m, m}=F_{1}^{1}$. We see easily also that $f\left(G_{m, m^{\prime}}\right)$ and $G_{m, m}$, intersect transversally, according to the formulas of local coordinate system of $G_{m, m^{\prime}}[15]$.

The general case for the Stiefel-Whitney class. Let $h: M \rightarrow G_{m, m^{\prime}}$ be a $C^{\infty}$ classifying of map $\eta$. Modifying $h$ if necessary, we can assume that $h$ is transversal to $F_{1}^{1}$. Then $h^{-1}\left(F_{1}^{1}\right)$ realizes the $m$-th Stiefel-Whitney class of $\eta$. Put $f^{\prime}=\tilde{h}^{-1} \circ f \circ h$, where $\tilde{h}$ is the lift of $h$. Then it follows immediately that $f^{\prime}(M)$ intersects transversally with $M$ at $h^{-1}\left(F_{1}^{1}\right)$.

For the Euler class, we need only to use $\widetilde{G}_{m, m^{\prime}}$ the oriented Grassmann 
manifold like we did in Proof of Theorem 2. We omit the details.

We can prove the following in the same way as Theorems $3^{\prime}, 4^{\prime}$.

Theorem 6. If $\operatorname{codim} V^{\prime}=1$, then $\alpha \cup \alpha^{\prime}$ is realized by an algebraic subvariety, and the set of singular points of the subvariety is contained in that of $V$. Here, if the coefficient ring is $\mathbb{Z}$, we assume moreover the compactness of $X$.

\section{§6. Affine Algebraic Structure of Vector Bundles}

Let $\eta: E \stackrel{\pi}{\longrightarrow} X$ be a topological vector bundle of rank $m$. We say that $\eta$ has an affine algebraic structure if $\eta$ is equivalent to an affine algebraic vector bundle. It is proved in [1] that if $X \approx S^{n}, \eta$ has an affine algebraic structure.

Conjecture. Assume that all Stiefel-Whitney classes of $\eta$ are realized by algebraic subvarieties. Then $\eta$ has an affine algebraic structure.

Theorem 7. If $m=1$, Conjecture is correct.

Proof. Let $V \subset X$ be an algebraic subvariety which realizes the StiefelWhitney class of $\eta$. By Theorem 3, we can assume the smoothness of $V$. Let $X^{\prime}$ be the desingularization of Hironaka of the algebraic closure of $X$ in $P^{n^{\prime}}(\boldsymbol{R})$ where $X \subset \mathbb{R}^{n^{\prime}}$, and $V^{\prime}$ be the algebraic closure of $V$ in $X^{\prime}$. Then there exists a line bundle $\eta^{\prime}$ over $X^{\prime}$ whose Stiefel-Whitney class is realized by $V^{\prime}$. Since line bundles over $X$ are determined by their Stiefel-Whitney classes, we can regard $\eta$ as the restriction of $\eta^{\prime}$ on $X$. Hence it is sufficient to find an affine algebraic structure of $\eta$. Therefore, from the beginning we assume that $X$ is compact.

Let $V_{0}=V, V_{1}, \ldots, V_{n}$ be distinct $C^{\infty}$ submanifolds of $X$ such that each $V_{i}$ is the image of $V$ under a $C^{\infty}$ diffeomorphism close to the identity. Using the transversality theorem, we reduct $V_{i}, i=1, \ldots, n$ to satisfy $\bigcap_{i=0}^{n} V_{i}=\phi$. Apply Theorem 3 to $V_{1}, \ldots, V_{n}$. Then we can assume also that $V_{l}$ are non-singular algebraic subvarieties. Let $V_{i j}$ denote the domain sandwiched in between $V_{i}$ and $V_{j}$ for each $i, j$. Put $U_{i}=X-V_{i}, i=0, \ldots, n$. Then $\left\{U_{i}\right\}$ is an open algebraic covering of $X$. If we have smooth rational functions $\psi_{i j}$ on $U_{i j}$ $=U_{i} \cap U_{j}$ for each $i, j$ such that

$$
\begin{aligned}
& \psi_{i j}=1, \psi_{i j} \psi_{j k}=\psi_{i k}, \text { and } \\
& \psi_{i j}\left\{\begin{array}{lll}
>0 & \text { on } & U_{i j}-V_{i j} \\
<0 & \text { on } & V_{i j},
\end{array}\right.
\end{aligned}
$$


then there exists an algebraic line bundle over $X$ which is equivalent to $\eta$. Moreover, if the bundle has a complexification, it is affine (see, e.g., [13]).

For each $i=1, \ldots, n$, we can construct a smooth rational function $f_{i}$ on $X$ in the same way as in Proofs of Theorems 3, 4 such that

(i) $f_{i}^{-1}(0)=V_{0} \cup V_{i}$,

(ii) $f_{i}<0$ in $V_{0_{i}}$,

(iii) $f_{i}$ is regular at $\left(V_{0}-V_{i}\right) \cup\left(V_{i}-V_{0}\right)$, and

(iv) for any $x \in V_{0} \cap V_{i}$, the germ of $f_{i}$ at $x$ is the product of two regular function germs.

Put $\psi_{i j}=f_{i} / f_{j}$. Then $\psi_{i j}$ satisfy the conditions above and have complexifications. Hence Theorem is proved.

\section{References}

[1] Benedetti, R. and Tognoli, A., On real algebraic vector bundles, Bull. Sci. Math., 104 (1980), 89-112.

[2] Bochnak, J., Kucharz, W. and Shiota, M., The divisor class groups of some rings of global real analytic, Nash or rational regular functions, to appear.

[ 3 ] Borel, A. and Haefliger, A., La classe d'homologie fondamental d'un espace analytique, Bull. Soc. Math. France, 89 (1961), 461-513.

[4] Grothendieck, A., La théorie des classes de Chern, Bull. Soc. Math. France, 86 (1958), $137-154$.

[ 5 ] Milnor, J. W. and Stasheff, J. D., Characteristic classes, Ann. of Math. Studies, 76, Princeton Univ. Press, 1974.

[6] Morimoto, H., Realization of Chern classes by subvarieties with certain singularities, Nagoya Math. J., 80 (1980), 49-74.

[7] Palais, R., Equivariant, real algebraic differential topology, Part I smoothness categories and Nash manifolds, Notes Brandeis Univ., 1972.

[ 8 ] Shiota, M., Sur la factorialité de l'anneau des fonctions lisses rationnelles, C.R.Acad. Sc. Paris, 292 (1981), 67-70.

[9] Shiota, M., Equivalence of differentiable functions, rational functions and polynomials, Ann. Inst. Fourier, 32 (1982).

[10] Suzuki, H., On the realization of the Stiefel-Whitney characteristic classes by submanifolds, Tôhoku Math. J., 10 (1958), 91-115.

[11] Thom, R., Quelques propriétés globales des variétés différentiables, Comm. Math. Helv. 28 (1954), 17-86.

[12] Tom, R., Un lemme sur less applications différentiables, Bol. Soc. Mat. Mexicana, 1 (1956), 59-71.

[13] Tognoli, A., Algebraic geometry and Nash functions, Academic Press, 1978.

[14] Whitney, H., Tangents to an analytic variety, Ann. of Math., 81 (1965), 496-549.

[15] Wu, W. T., Sur les classes charactéristiques des structures fibrées sphériques, Act. Sci. Ind., 1183, Hermann, 1952.

Added in proof: The author was informed that some of his results were obtained also by R. Benedetti and A. Tognoli (Remarks and counterexamples in real algebraic vector bundles and cycles, to appear). 